
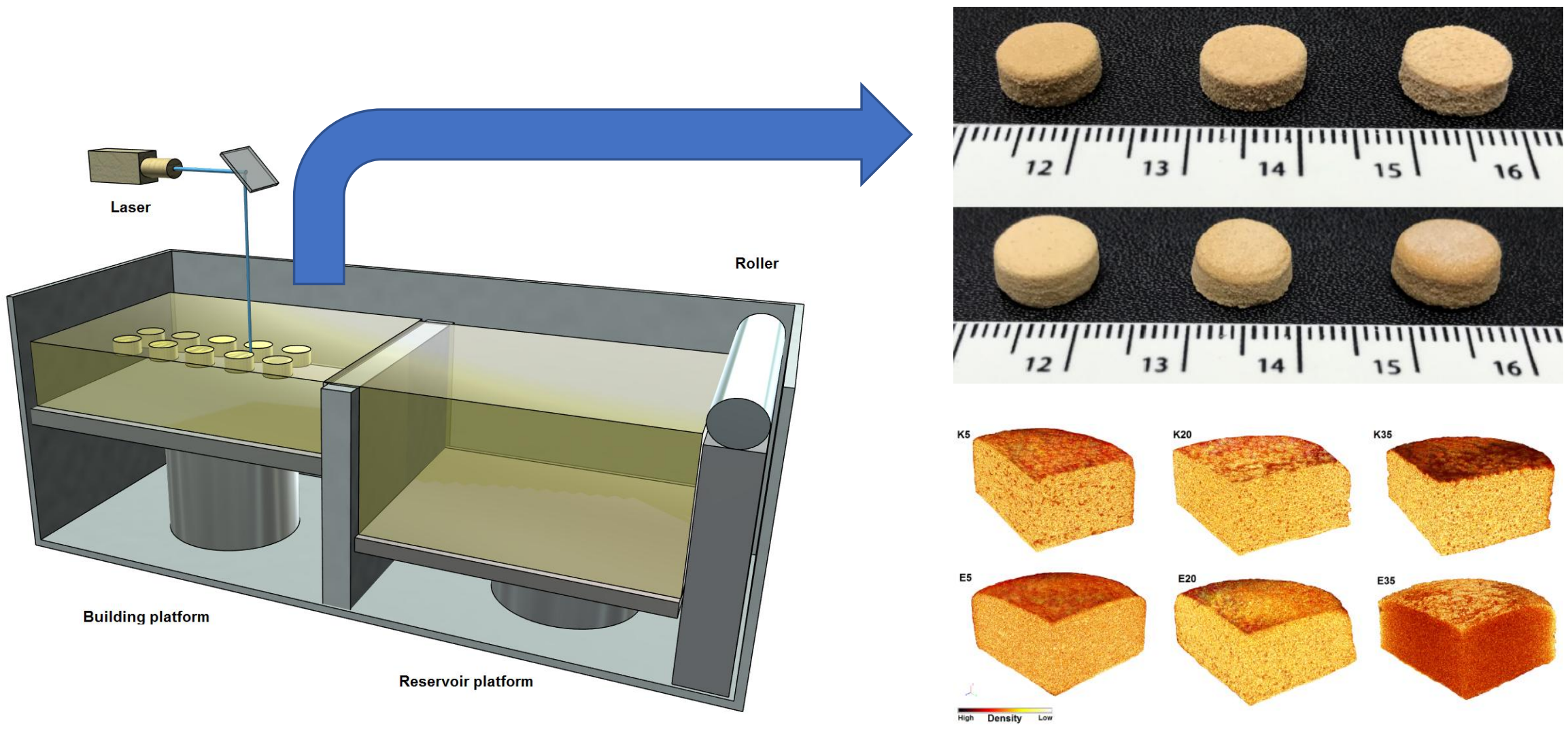


\section{Selective Laser Sintering (SLS) 3D printing of medicines}

2

3 Fabrizio Fina ${ }^{1 *}$, Alvaro Goyanes ${ }^{2 *}$, Simon Gaisford ${ }^{1,2}$, Abdul W. Basit $^{1,2}$ 4

$5{ }^{1}$ UCL School of Pharmacy, University College London, 29-39 Brunswick Square, London, 6 WC1N 1AX, UK

$7 \quad{ }^{2}$ FabRx Ltd., 3 Romney Road, Ashford, Kent, TN24 ORW, UK

8

9 10

11 Corresponding author:

12 Abdul W. Basit

13 a.basit@ucl.ac.uk

14 Tel: 02077535865 
Selective laser sintering (SLS) 3-dimensional printing is currently used for industrial manufacturing of plastic, metallic and ceramic objects. To date there are no reports on the use of SLS to fabricate oral drug loaded products, hence, However, the extreme printing conditions (temperatures $>1000^{\circ} \mathrm{C}$ and high-energy lasers $>250 \mathrm{~W}$ ) used in these fields have precluded its use in the pharmaceutical sector. tThe aim of this work was to explore the suitability of SLS printing for manufacturing medicines. Two thermoplastic pharmaceutical grade polymers, Kollicoat IR (75\% polyvinyl alcohol and $25 \%$ polyethylene glycol copolymer) and Eudragit L100-55_50\% methacrylic acid and 50\% ethyl acrylate copolymer), with immediate and modified release characteristics respectively, were selected to investigate the versatility of a new desktop-SLS printer. Each polymer was investigated with three different drug loadings of paracetamol (acetaminophen) $(5,20$ and 35\%). To aid the sintering process, To improve the sintering process, $3 \%$ Candurin $^{\circledR}$ gold sheen colourant-was added to each of the powdered formulations. In total ${ }_{2}$ six solid formulations were successfully printed; the printlets were robust ${ }_{2}$ and no evidence of drug degradation was observed. In biorelevant bicarbonate dissolution media, the Kollicoat formulations showed $\mathrm{pH}_{-}$ independent release characteristics, with the rate of release dependent on the drug content. In the case of the Eudragit formulations, these showed $\mathrm{pH}_{-}-$dependent, modified--release profiles independent of drug loading, with complete release being achieved over 12 hours. In conclusion, this work has demonstrated that SLS is a versatile and practical 3D printing technology which can be applied to the pharmaceutical field, thuserefore widening the armamentarium number of 3D printing technologies available for the to manufacture of modern medicines. 


\section{Introduction}

3-Dimensional printing (3DP) is an additive manufacturing technology withwhich finds applications in myriadany different fields from including-medical device manufacturing to, aeronautics, robotics, electronics, industrial goods and even the food industry-(Sculpteo, 2017)_(Barnatt, 2013). 3DP for the fabrication of medicines has come to the fore in recent years, specifically for its revolutionary uses in personalised dose and dimension-specific dosage form printing.- and it has been anticipated to have a revolutionary impact on healthcare. The replacement of conventional drug manufacture and distribution could provide patients with personalized polypills fabricated at the point of care to reduce cost and enhance therapy adherence (Choonara et al., 2016).

The first attempt at using 3DP technology in pharmaceuticals dates back to 1996 (Wu et al., 1996), whereby a powder bed 3D printer (PB) was employed to produce a 3D solid form containing drug. PB technology, similarly to the widespread inkjet desktop printers that use an ink (black or colour) to print onto a paper sheet, selectively deposits a liquid binder material across a powder bed. The process is repeated layer-by-layer to fabricate a 3D object. This technology has been adopted to manufacture Spiritam ${ }^{\circledR}$, the first FDA--approved 3D printed drug product, that came into the market in 2016 for the treatment of epilepsy (Aprecia-Pharmaceuticals, 2016).

An alternative 3DP technique termed stereolithograpy (SLA) has recently been used to manufacture printlets, containing either paracetamol or 4-ASA (Wang et al., 2016), and antiacne masks (Goyanes et al., 2016a). SLA technology uses a laser to solidify a photopolymerizable polymer solution containing drug. Advantages of this technology include production of high resolution objects at room temperature. However, limitations such as carcinogenic risk of the photopolymerizing material limits its short-term implementation and demands further investigations.

Fused_-deposition modeling (FDM) has been the most employed 3DP technology to date, due to it being inexpensive and easy to use. Here, previously extruded polymer-based filaments are forced through heated nozzles turning them into semi-liquid materials that are selectively deposited onto a printing platform layer-by-layer (Goyanes et al., 2014). For oral medicines, FDM printing was first used to manufacture polyvinyl alcohol (PVA)--based printlets, incorporating different drugs (Goyanes et al., 2014; Goyanes et al., 2015a; Goyanes et al., 2015b) with different geometries (Goyanes et al., 2015f) and drug distribution (Goyanes et al., 2015g). More recently, several pharmaceutical grade polymers have been reported to be suitable formulation candidates for FDM printing (Melocchi et al., 
2016; Pietrzak et al., 2015), providing oral formulations with fast-rapid drug release profiles (Okwuosa et al., 2016) and enteric properties (Goyanes et al., 2017; Okwuosa et al., 2017). However, limitations of FDM 3DP include use of high printing temperatures $\left(>120^{\circ} \mathrm{C}\right)$, which may induce drug degradation, and a relatively low resolution of the printed objects.

Selective laser sintering (SLS) is an industrial 3DP technology that uses a powder bed to build up the 3D object, similarly to PB. However, instead of using a spray solution, SLS uses a laser to bind the powder particles together. During the printing process, the laser is directed to draw a specific pattern onto the surface of the powder bed. Once the first layer is completed, a roller distributes a new layer of powder on top of the previous one. The object is built layer-by-layer, which will-is then be recovered from underneath the powder bed. Advantages of SLS technology include the fact that it a solvent-free process and offers faster production as compared to $\mathrm{PB}_{1}$ which instead requires the printed object to be left for up to 48 hours to allow the solvent to evaporate (Rowe et al., 2000; Yu et al., 2009; Yu et al., 2007). Compared to FDM, SLS is a one-step process that does not require the prior production of suitable filaments by hot melt extrusion (Goyanes et al., 2015b; Goyanes et al., 2015g; Okwuosa et al., 2017) and produces objects of higher resolution due to the laser precision. However, commonly used materials are powdered forms of plastics, ceramics and metal alloys that require high temperatures $\left(1000^{\circ} \mathrm{C}\right.$ andor more higher) and high-energy lasers (250W and higheror more) to be sintered (Vrancken et al., 2012)..-.These harsh printing conditions have hindered entry of this technology into the pharmaceutical field. It is widely recognised that the high--energy input of the laser may degrade drugs if they are used as the starting material (Alhnan et al., 2016; Yu et al., 2008). For these reasons, the sole use of SLS printing in the medical field has been limited to either tissue engineering scaffolds (Partee et al., 2006) or drug delivery devices where the drug was included after the printing process (Cheah et al., 2002; Leong et al., 2006). So far, no studies have been reported investigating the production of drug loaded formulations using SLS.

The aim of this study was to explore SLS printing as a suitable 3DP technology for the preparation of drug loaded oral dosage forms using pharmaceutical grade excipients. The versatility of the printer was evaluated using two different polymers commonly used to manufacture immediate and modified release oral formulations.

\section{Materials and methods}

Paracetamol USP grade (Sigma-Aldrich, UK) was used as a model drug (MW 151.16, solubility at $37^{\circ} \mathrm{C}: 21.80 \mathrm{~g} / \mathrm{L}$ (Yalkowsky and $\left.\mathrm{He}, 2003\right)$ ). 
Kollicoat IR (BASF, UK) is a graft copolymer composed of $75 \%$ polyvinyl alcohol units and $25 \%$ polyethylene glycol units with a molecular weight of approximately 45,000 Daltons that is mainly used for instant release coatings (BASF, 2017). Eudragit L100-55, a copolymer of methacrylic acid and ethyl acrylate (1:1 ratio) a methacrylic acid co-polymer that dissolves at pH above -5.5 and above (Evonik, 2017), was donated by Evonik, UK. Candurin ${ }^{\circledast}$ Gold Sheen was purchased kindly donatedfrom-by Azelis, UK. The salts for preparing the buffer dissolution media were purchased from VWR International Ltd., UK.

\subsection{Printing process}

For each formulation $100 \mathrm{~g}$ of a mixture of drug and excipients were blended using a mortar and pestle (Table 1). $3 \%$ of Candurin $^{\oplus}$ Gold Sheen colourant-was added to the formulations as an absorbent material to enhance energy absorption from the laser and aid allow printability.

Powder mixtures were then transferred to a-Desktop SLS printer (Sintratec Kit, AG, Brugg, Switzerland) to fabricate the oral dosage formulations. AutoCAD 2014 (Autodesk Inc., USA) was used to design the templates of the cylindrical printlets $(10 \mathrm{~mm}$ diameter $\times 3.6 \mathrm{~mm}$ height). 3D models were exported as a stereolithography (.stl) file into 3D printer Sintratec central software Version 1.1.13.

Powder in the platform reservoir $(150 \times 150 \times 150 \mathrm{~mm})$ of the printer was moved by a sled to a building platform $(150 \times 150 \mathrm{~mm})$ creating a flat and homogeneously distributed layer of powder. The printer was warmed up for at least one hour to allow the heat to be thoroughly distributed inside the printer including the whole reservoir of powder. Two different temperatures were chosen and kept the same for all formulations in this study: a chamber temperature $\left(90^{\circ} \mathrm{C}\right)$, indicating the temperature inside the printer; and a surface temperature $\left(110^{\circ} \mathrm{C}\right)$, indicating the surface temperature of the powder bed in the building platform. The printing process started with the activation of a When the heating process was completed, the $2.3 \mathrm{~W}$ blue diode laser $(445 \mathrm{~nm}$ ) was activated-(laser scanning speed $90 \mathrm{~mm} / \mathrm{sec}$ ) to sinter the powder on to the building platform in a certain pattern based on the .STL file. At this point, the reservoir platform moved up, the building platform moved down and the sled distributed a thin layer of powder on top of the previous layer. This process was repeated layer-by-layer until the object was completed. Printlets were then removed from the powder bed and the excess power was brushed off. Five-Ten printlets were printed at the same time for each formulation.

\subsection{Powder spectrophotometer analysis}


UV-Vis-NIR spectrophotometer Shimadzu UV-2600 was employed to measure the absorbance in the solid state of the drug and/or excipient-sand/or colourant material. Absorbance at wavelengths between $220-1400 \mathrm{~nm}$, was measured at room temperature (approximately $25^{\circ} \mathrm{C}$ ) using an integrating sphere as "Diffuse Reflectance Accessory (DRA)". Here $0.15 \mathrm{~g}$ of material to be evaluated (polymer, drug, colourant_or mixtures of themthese) was blended with $0.5 \mathrm{~g}$ of barium sulphate and compressed to form a barium sulphate disk that is introduced in the spectrophotometer for analysis.

\subsection{Thermal analysis}

Differential scanning calorimetry (DSC) and thermogravimetric analysis (TGA)-wasere used to characterise the powders and the drug loaded printlets. DSC measurements were performed with a Q2000 DSC (TA instruments, Waters, LLC, USA) at a heating rate of $10^{\circ} \mathrm{C} / \mathrm{min}$. Calibration for cell constant and enthalpy was performed with indium ( $\mathrm{Tm}=$ $156.6^{\circ} \mathrm{C}, \Delta \mathrm{Hf}=28.71 \mathrm{~J} / \mathrm{g}$ ) according to the manufacturer instructions. Nitrogen was used as a purge gas with a flow rate of $50 \mathrm{~mL} / \mathrm{min}$ for all the experiments. Data were collected with TA Advantage software for $Q$ series (version 2.8.394), and analysed using TA Instruments Universal Analysis 2000. All melting temperatures are reported as extrapolated onset unless otherwise stated. TA aluminium pans and lids (Tzero) were used with an average sample mass of $8-10 \mathrm{mg}$.

For TGA analysis, samples were heated at $10^{\circ} \mathrm{C} / \mathrm{min}$ in open aluminium pans with a Discovery TGA (TA instruments, Waters, LLG, USA). Nitrogen was used as a purge gas with a flow rate of $25 \mathrm{~mL} / \mathrm{min}$. Data collection and analysis were performed using TA Instruments Trios software and \% mass loss and/or onset temperature were calculated.

\subsection{X-ray powder diffraction (XRPD)}

Discs of $23 \mathrm{~mm}$ diameter $\times 1 \mathrm{~mm}$ height made from the mixtures of drug and excipients were 3D printed and analysed. Samples of pure paracetamol and the mixtures were also analysed. The X-ray powder diffraction patterns were obtained in a Rigaku MiniFlex 600 (Rigaku, USA) using a Cu Ka X-ray source $(\lambda=1.5418 \AA)$. The intensity and voltage applied were $15 \mathrm{~mA}$ and $40 \mathrm{kV}$. The angular range of data acquisition was $3-60^{\circ} 2 \theta$, with a stepwise size of $0.02^{\circ}$ at a speed of $5 \% \mathrm{~min}$.

\subsection{Printlets $\underline{\text { Ccharacterisation of the printlets }}$}

\subsubsection{Determination of printlet morphology}


The diameter and thickness of the printlets were measured using a digital calliper. Pictures were taken with a Nikon CoolpixS6150 with the macro option of the menu.

\subsubsection{Determination of printlet strength}

The crushing strength of ten printlets of each type was measured using a traditional tablet hardness tester TBH 200 (Erweka GmbH, Heusenstamm, Germany), whereby an increasing force is applied perpendicular to the printlet axis to opposite sides of a printlet until the printlet fractures.

\subsubsection{Determination of printlet friability}

Approximately $6.5 \mathrm{~g}$ of printlets were weighed and placed into the drum of a Friability Tester Erweka type TAR 10 (Erweka $\mathrm{GmbH}$, Heusenstamm, Germany). The drum was then rotated at $25 \mathrm{rpm}$ for $4 \mathrm{~min}$ and the sample re-weighed. The friability of the sample is given in terms of weight loss, expressed as a percentage of the original sample weight.

\subsubsection{Scanning Electron Microscopy (SEM)}

Surface and cross-section images of the printlets were taken with a scanning electron microscope (SEM, JSM-840A Scanning Microscope, JEOL GmbH, Germany). All samples for SEM testing were coated with carbon ( 30-40 nm).

\subsubsection{X-ray Micro Computed Tomography (Micro-CT)}

A high-resolution X-ray micro computed tomography scanner (SkyScan1172, BrukermicroCT, Belgium) was used to $3 \mathrm{D}$ visualize the internal structure, density and porosity of the printlets. All oral formulations were scanned using no filter with a resolution of $2000 \times 1048$ pixels. 3D imaging was performed by rotating the object through $180^{\circ}$ with steps of $0.4^{\circ}$ and 4 images were recorded for each of those. The total acquisition time was about 25 mins per sample. Image reconstruction was performed using NRecon software (version 1.7.0.4, Bruker-microCT). 3D model rendering and viewing were performed using the associate program CT-Volume (CTVol version 2.3.2.0) software. The collected data was analysed using the software CT Analyzer (CTan version 1.16.4.1). Different colours were used to indicate the density of the printlets. Porosity values were calculated using the 3D analysis in the morphometry preview (200 layers were selected at the central part of the printlet as area of interest and analysed).

\subsubsection{Determination of Drug Content}


Three individual printlets of each formulation A printlet (approximately $0.2 \mathrm{~g}$ )-wereas placed in a-separate volumetric flask $\underline{s}$ with deionized water $(250 \mathrm{ml})$. - In the case of the Eudragit-based printlets, 3 drops of $5 \mathrm{~N} \mathrm{NaOH}$ were added to the flasks to increase the $\mathrm{pH}$ in order to dissolve the polymers under magnetic stirring until complete dissolution. Samples of solution were then filtered through $0.45 \mathrm{~mm}$ filters (Millipore Ltd., Ireland) and the concentration of drug determined with HPLC (Hewlett Packard 1050 Series HPLC system, Agilent Technologies, UK). The validated high performance liquid chromatographic assay entailed injecting $20 \mathrm{~mL}$ samples for analysis using a mobile phase, consisting of methanol (15\%) and water (85\%), through an Ultra C8 $5 \mu \mathrm{m}$ column, $25 \times 4.6 \mathrm{~mm}$ (Restek, USA) maintained at $40^{\circ} \mathrm{C}$. The mobile phase was pumped at a flow rate of $1 \mathrm{~mL} / \mathrm{min}$ and the eluent was screened at a wavelength of $247 \mathrm{~nm}$. All measurements were made in triplicate.

\subsubsection{Dynamic dissolution testing conditions}

Drug dissolution profiles for the formulations were obtained with a USP-II apparatus (Model PTWS, Pharmatest, Germany): 1) the formulations were placed in $750 \mathrm{~mL}$ of $0.1 \mathrm{M}$ $\mathrm{HCl}$ for $2 \mathrm{~h}$ to simulate gastric residence time, and then 2) transferred into $950 \mathrm{~mL}$ of modified Hanks (mHanks) bicarbonate physiological medium for $35 \mathrm{~min}$ (pH 5.6 to 7); 3) and then in modified Krebs buffer $(1000 \mathrm{ml})(\mathrm{pH} 7$ to 7.4 and then to 6.5$)$. The modified Hanks buffer based dissolution medium (Liu et al., 2011) (136.9 mM NaCl, $5.37 \mathrm{mM} \mathrm{KCl,} 0.812 \mathrm{mM}$ $\mathrm{MgSO}_{4} .7 \mathrm{H}_{2} \mathrm{O}, 1.26 \mathrm{mM} \mathrm{CaCl}, 0.337 \mathrm{mM} \mathrm{Na}_{2} \mathrm{HPO}_{4} .2 \mathrm{H}_{2} \mathrm{O}, 0.441 \mathrm{mM} \mathrm{KH} \mathrm{PO}_{4}, 4.17 \mathrm{mM}$ $\mathrm{NaHCO}_{3}$ ) forms an in-situ modified Kreb's buffer (Fadda et al., 2009) by addition of $50 \mathrm{~mL}$ of pre-Krebs solution ( $400.7 \mathrm{mM} \mathrm{NaHCO}_{3}$ and $6.9 \mathrm{mM} \mathrm{KH}_{2} \mathrm{PO}_{4}$ ) to each dissolution vessel.

The formulations were tested in the small intestinal environment for $3.5 \mathrm{~h}(\mathrm{pH} 5.6$ to 7.4), followed by pH 6.5 representing the colonic environment (Fadda et al., 2009; Goyanes et al., 2015c; Goyanes et al., 2015d; Liu et al., 2011). The medium is primarily a bicarbonate buffer in which bicarbonate $\left(\mathrm{HCO}_{3}{ }^{-}\right)$and carbonic acid $\left(\mathrm{H}_{2} \mathrm{CO}_{3}\right)$ co-exist in an equilibrium, along with $\mathrm{CO}_{2}$ (aq) resulting from dissociation of the carbonic acid. The $\mathrm{pH}$ of the buffer is controlled by an Auto pH System ${ }^{\mathrm{TM}}$ (Merchant et al., 2012; Merchant et al., 2014), which consists of a pH probe connected to a source of carbon dioxide gas ( $\mathrm{pH}$-reducing gas), as well as to a supply of helium ( $\mathrm{pH}$-increasing gas), controlled by a control unit. The control unit is able to provide a dynamically adjustable $\mathrm{pH}$ during testing (dynamic conditions) and to maintain a uniform $\mathrm{pH}$ value over the otherwise unstable bicarbonate buffer $\mathrm{pH}$.

The paddle speed of the USP-II was fixed at $50 \mathrm{rpm}$ and the tests were conducted at 37 $+/-0.5{ }^{\circ} \mathrm{C}(\mathrm{n}=3)$. Sample of the dissolution media $(1 \mathrm{~mL})$ was withdrawn every hour and the drug concentrations were determined by HPLC to calculate the percentage of drug released from the formulations. 


\section{Results and discussion}

Two thermoplastic excipients frequently used in hot melt extrusion, Kollicoat IR (Kollicoat) and Eudragit L100-55_(Eudragit), were initially tested to evaluate their printability by SLS 3DP, alone or in combination with $5 \%$ paracetamol. However, in the preliminary experiments, the laser did not lead to sintering of the powders.have-any effect on the polymer powders.

SLS printers use a unique binding thermal process to connect the powder particles together (Shirazi et al., 2015). The laser is aimed to draw a specific pattern on the powder bed that increases the local temperature. If the temperature reaches a value between the melting temperature $\left(T_{m}\right)$ of the material and $T_{m} / 2$, a solid-state sintering will happen that partially fuses the powder particles together. If the temperature overcomes the $T_{m}$, a full melting occurs producing stronger objects with reduced porosity, as the molten polymer will infiltrate into the voids between the powder particles.

The ideal temperature can be reached by adjusting the internal temperature of the printer and the laser scanning speed. By reducing the laser scanning speed, a longer interaction time between the powder particles and the laser beam leads to a higher transmission of energy producing denser objects. On the contrary, upon increasing the laser scanning speed, less energy is transmitted leading to the production of weaker and more porous objects (Shirazi et al., 2015).

Since even a slower laser scanning speed did not produce any sintering effect on the powder bed it was supposed-hypothesized the absence of interaction between the laser beam and the powder. that the powder absorbance was not adequate. The evaluation of the absorbance characteristics for the two polymers and paracetamol was obtained using a Shimadzu UV-3600 Plus UV-VIS-NIR spectrophotometer with an integrating sphere. The absorbance values checked at the same wavelength of the blue diode laser provided with the printer $(445 \mathrm{~nm})$ were all close to the baseline indicating that the selected excipients did not absorb the laser light precluding the sintering process. Candurin ${ }^{\circledR}$ gold sheen an Since the laser is in the blue spectrum, the maximum absorbance occurs with its complementary colours, orange or yellow. Therefore, a GRAS-approved pharmaceutical excipient colorant used for coating of tablets (Candurin ${ }^{\circledR}$ gold sheen) was selected and included into the drug and polymer mixture at $3 \% \mathrm{w} / \mathrm{w}$ and showeddue to its -a-good degree of absorbance at 445 $\mathrm{nm}$-suggesting a possible laser sintering.

In contrast to the previous tests without colourant,By including the colourant Candurin ${ }^{\circledR}$ gold sheen absorbent materialin the mixtures, the powder particles in the area where the laser was aimed were sintered and well connected. For this study, a chamber temperature of 
$90{ }^{\circ} \mathrm{C}$, a surface temperature of $110{ }^{\circ} \mathrm{C}$ and a laser scanning speed of $90 \mathrm{~mm} / \mathrm{sec}$ were found to be suitable parameters which were maintained throughout printing of all the formulations. The manufacture of solid dosage forms was successfully achieved and the printing process was then repeated to obtain six different formulations containing $3 \% \mathrm{w} / \mathrm{w}$ Candurin ${ }^{\oplus}$ gold sheenabsorbent materialcolourant, based on either Kollicoat IR or Eudragit L100-55, each with three different drug loadings $(5,20$ and $35 \% \mathrm{w} / \mathrm{w})$ (Table 1). The formulations produced were all smooth and yellow in colour (Figure 1).

The printlet strength data for Kollicoat formulations exceeded the highest value that the equipment could measure because the printlets did not break but they deformed. For Eudragit formulations, crushing strength values ranged between $284 \mathrm{~N}$ and $414 \mathrm{~N}$ (Table 2). Friability of all the formulations was less than 1\%, complying with the US pharmacopeia requirement for uncoated tablets, making them suitable for handling and packing (USP, 2017).

X-ray micro-CT was employed to calculate closed and open porosity of the printlets (Table 2) and to visualise their internal structures (Figure 2). Kollicoat formulations showed similar total porosity (closed + open porosity) values for all three drug loadings, whereas the Eudragit formulations showed a clear reduction in total porosity with increasing drug content. Additionally, the higher the drug content, the more the closed porosity (in E5 there were almost no closed pores, while in E35 more than $80 \%$ of the total porosity was made up of closed pores) suggesting that the material was more sintered or even melted. Different colours were given depending on the density levels. All printlets showed similar density values except for E35 that which was denser, in part due to explained by its very low porosity and high crushing strength (Table 2 ).

SEM images of the printlets provided a visual confirmation of the porosity and the strength values discussed above (Table 2). Kollicoat formulations images show a sintering process for $\mathrm{K} 5$ (limited molten areas) that becomes a combination of sintering/melting (more molten areas are visible) for K20 and an almost total melting for K35 leading to stronger printlets. The same trend is clearly visible for Eudragit formulations, where the single spherical polymer particles can be easily distinguished in E5 while they become indistinguishable for the $35 \%$ loaded printlets. Additionally, it is clearly visible in E5 as the sintering process, created mainly open pores, while E35, being effectively melted, has mainly closed pores.

Since the laser degradation of the drug was a main concern of the feasibility study, the drug content of the printlets were evaluated. All the values were close to the theoretical drug loading $(5,20$ and $35 \%)$ and no other peaks other than paracetamol were present in the 
HPLC chromatograms, indicating that the drug degradation did not take place $\theta$ ccur-during printing (Table 2).

DSC and X-ray analyses of the drug, mainindividual polymers, mixed materials before printing and printlets were performed to explore the drug phase state and to which degree the drug is incorporated into the polymers (Figures 4 and 5). DSC data shows that paracetamol raw material melts at around $168^{\circ} \mathrm{C}$ indicative of form I (Goyanes et al., 2015e). The DSC data of the printlets showed no evidence of melting at around $168^{\circ} \mathrm{C}$, indicating that the drug is either molecularly dispersed within the polymer matrix as a solid dispersion or that the drug is dissolved into the polymer during the temperature increase within the DSC process. It is possible to observe an endotherm attributed to the melting of paracetamol in the physical mixture for all the polymers even at the lowest drug content, indicating that part of the drug is in the crystalline form.

In accordance with the DSC, X-ray diffractograms showed semi-crystalline patterns with the presence of the characteristic paracetamol peaks in all the physical mixtures.

X-ray powder diffractograms for Kollicoat printlets show paracetamol peaks and the patterns of the polymers are similar to those of the physical mixture (Figure 5). This confirms that at least part of the drug is present in a crystalline form.

Diffractograms of the Eudragit printlets do not show any paracetamol peaks at any level of drug loading (Figure 5). This confirms that the drug in the Eudragit printlet is present in an amorphous phase within the polymer matrix, as observed in the DSC. Paracetamol, which has a high melting point of about $168^{\circ} \mathrm{C}$, may have dissolved in the molten polymer during the printing process.

Figures 6 and 7 show the dissolution characteristics of all the formulations. Printlets were tested in the dynamic in vitro model, which simulates gastric and intestinal conditions of the gastrointestinal tract (Goyanes et al., 2015c). A reduction in the size of the formulations during the dissolution tests was observed, indicating that erosion processes may be involved in modulating drug release from these 3DP formulations, as previously suggested (Goyanes et al., 2016b).

The dissolution profiles of Kollicoat printlets show that drug release commenced during the gastric phase and was not affected by the $\mathrm{pH}$ of the media, indicating that the formulations were $\mathrm{pH}$ independent (Figure 6).

K5 reached over $80 \%$ of drug release in about 30 mins whereas K20 and K35 reached the same value in about $2 \mathrm{~h}$ and $5 \mathrm{~h}$, respectively (Figure 6). A complete drug release was achieved in about $2 \mathrm{~h}$ for $\mathrm{K} 5$, compared to about $10 \mathrm{~h}$ for both $\mathrm{K} 20$ and $\mathrm{K} 35$. As previously 
seen (Figures 2 and 3, Table 2), an increasing drug content leads to more sintered/melted and less porous printlets that will require longer time to dissolve. For all three Kollicoat formulations, after $24 \mathrm{~h}$ dissolution test, a small residue of printlet was found in the vessels; however, the drug was already entirely released.

The dissolution results for Eudragit printlets showed some drug release in the gastric phase (acidic medium) that increased during the intestinal phase (biorelevant bicarbonate buffers), being dependent on the nature or $\mathrm{pH}$ of the media (Figure 7).

Eudragit L100-55 is an enteric polymer, however some paracetamol was released during the first $2 \mathrm{~h}$ (acidic environment) for all three formulations. This may be as a consequence of their matrix structure, whereby the drug is evenly distributed including at the external surface, permitting release once in contact with the dissolution media. As expected, dissolution data after $2 \mathrm{~h}$ showed about 18\%, 14\% and 6\% paracetamol release for E5, E20 and E35, respectively. These values are correlated with the open porosity of the printlets (Table 2); E5 is highly porous and only $0.1 \%$ of its total porosity is due to closed pores, allowing the acidic media to come into contact with a large surface area of the printlet. Conversely, E35 has a very low porosity that is mainly due to closed pores inside the printlet, limiting the surface in contact with the media and thus the drug release.

After the first $2 \mathrm{~h}$, all three formulations started to release faster in intestinal conditions at $\mathrm{pH} 5.5$ and above with the right $\mathrm{pH}$ threshold (above pH 5.5)-leading to a complete dissolution in about $12 \mathrm{~h}$. However, in the case of E5, drug release slowed down the release of drug-in colonic conditions (after 5h 30 mins') probably-presumably because the formulation was composed of $92 \% \mathrm{w} / \mathrm{w}$ enteric polymer, which was likely to be more affected by the reduction in $\mathrm{pH}$, compared to $\mathrm{E} 20$ and $\mathrm{E} 35_{-}$-

Interestingly, the overall release from the Eudragit printlets fabricated using SLS at three different drug loadings was analogue-very similar. The samesimilar release profile was for Eudragit formulations is-explained by a proportionally stronger sintering/melting effect on increased drug loaded printlets, as previously discussed (Figures 2 and 3, Table 2). This is different to FDM printlets that dissolved proportionally faster with an increased drug content (Goyanes et al., 2017; Goyanes et al., 2016b; Goyanes et al., 2015g). The same release profile for Eudragit formulations is explained by a proportionally stronger sintering/melting effect on increased drug loaded printlets, as previously discussed (Figures 2 and 3 , Table 2). Eudragit formulations might then provide a platform that allows to maintain the same release profile during a therapy with a progressive modulation of the drug dosage. 
Overall, the dissolution data shows the versatility of SLS printing to produce medicines

403 with different pharmaceutical polymers release profiles-and different drug loadings. More 404 importantly, since no drug degradation was detected during this study, this work opens up 405 the SLS 3DP technology to further investigation in the pharmaceutical field.

406

407

\section{Conclusion}

408

In this proof_-of_-concept study, a desktop_SLS printer was used to manufacture oral medicines using pharmaceutical grade excipients without degradation of the drug. The versatility of SLS technology has been demonstrated with the successful manufacture of immediate_-release and modified--release formulations with three different drug loadings. This work demonstrates the potential of SLS 3DP to produce personalized medicines: adding SLS to the armamentarium widening the number-of 3DP technologies available for the commercial to manufacture of medicines.

416 


\section{References}

Alhnan, M.A., Okwuosa, T.C., Sadia, M., Wan, K.-W., Ahmed, W., Arafat, B., 2016. Emergence of 3D Printed Dosage Forms: Opportunities and Challenges. Pharmaceutical Research 33, 1817-1832.

Aprecia-Pharmaceuticals, 2016. What is ZipDose® Technology? https://www.spritam.com/\#/patient/zipdose-technology/what-is-zipdose-technology.

Barnatt, C., 2013. 3D Printing: the next industrial revolution. Barnatt, C., UK.

BASF, 2017. Kollicoat® ${ }^{\circledR}$ IR is one of our true multitalents for instant release. https://pharmaceutical.basf.com/en/Drug-Formulation/Kollicoat-IR.html.

Cheah, C.M., Leong, K.F., Chua, C.K., Low, K.H., Quek, H.S., 2002. Characterization of microfeatures in selective laser sintered drug delivery devices. Proceedings of the Institution of Mechanical Engineers, Part H: Journal of Engineering in Medicine 216, 369-383.

Choonara, Y.E., du Toit, L.C., Kumar, P., Kondiah, P.P., Pillay, V., 2016. 3D-printing and the effect on medical costs: a new era? Expert review of pharmacoeconomics \& outcomes research 16, 23-32.

Evonik, 2017. Polymers for enteric release. http://healthcare.evonik.com/product/healthcare/en/products/pharmaceutical-excipients/delayed-release/pages/default.aspx.

Fadda, H.M., Merchant, H.A., Arafat, B.T., Basit, A.W., 2009. Physiological bicarbonate buffers: stabilisation and use as dissolution media for modified release systems. Int. J. Pharm. 382, 56-60.

Goyanes, A., Buanz, A.B., Basit, A.W., Gaisford, S., 2014. Fused-filament 3D printing (3DP) for fabrication of tablets. Int J Pharm 476, 88-92.

Goyanes, A., Buanz, A.B.M., Hatton, G.B., Gaisford, S., Basit, A.W., 2015a. 3D printing of modified-release aminosalicylate (4-ASA and 5-ASA) tablets. European Journal of Pharmaceutics and Biopharmaceutics 89, 157-162.

Goyanes, A., Chang, H., Sedough, D., Hatton, G.B., Wang, J., Buanz, A., Gaisford, S., Basit, A.W., 2015b. Fabrication of controlled-release budesonide tablets via desktop (FDM) 3D printing. International Journal of Pharmaceutics 496, 414-420.

Goyanes, A., Det-Amornrat, U., Wang, J., Basit, A.W., Gaisford, S., 2016a. 3D scanning and $3 D$ printing as innovative technologies for fabricating personalized topical drug delivery systems. Journal of Controlled Release 234, 41-48.

Goyanes, A., Fina, F., Martorana, A., Sedough, D., Gaisford, S., Basit, A.W., 2017. Development of modified release 3D printed tablets (printlets) with pharmaceutical excipients using additive manufacturing. International Journal of Pharmaceutics, https://doi.org/10.1016/i.ijpharm.2017.1005.1021.

Goyanes, A., Hatton, G.B., Basit, A.W., 2015c. A dynamic in vitro model to evaluate the intestinal release behaviour of modified-release corticosteroid products. J. Drug Deliv. Sci. Tec. 25, 36-42. 
Goyanes, A., Hatton, G.B., Merchant, H.A., Basit, A.W., 2015d. Gastrointestinal release behaviour of modified-release drug products: Dynamic dissolution testing of mesalazine formulations. Int. J. Pharm. 484, 103-108.

Goyanes, A., Kobayashi, M., Martinez-Pacheco, R., Gaisford, S., Basit, A.W., 2016b. Fusedfilament 3D printing of drug products: Microstructure analysis and drug release characteristics of PVA-based caplets. Int J Pharm 514, 290-295.

Goyanes, A., Martinez, P.R., Buanz, A., Basit, A., Gaisford, S., 2015e. Effect of geometry on drug release from 3D printed tablets. Int. J. Pharm. 494, 657-663.

Goyanes, A., Robles Martinez, P., Buanz, A., Basit, A.W., Gaisford, S., 2015f. Effect of geometry on drug release from 3D printed tablets. Int J Pharm 494, 657-663.

Goyanes, A., Wang, J., Buanz, A., Martinez-Pacheco, R., Telford, R., Gaisford, S., Basit, A.W., 2015g. 3D Printing of Medicines: Engineering Novel Oral Devices with Unique Design and Drug Release Characteristics. Molecular Pharmaceutics 12, 4077-4084.

Leong, K.F., Chua, C.K., Gui, W.S., Verani, 2006. Building Porous Biopolymeric Microstructures for Controlled Drug Delivery Devices Using Selective Laser Sintering. The International Journal of Advanced Manufacturing Technology 31, 483-489.

Liu, F., Merchant, H.A., Kulkarni, R.P., Alkademi, M., Basit, A.W., 2011. Evolution of a physiological $\mathrm{pH} 6.8$ bicarbonate buffer system: Application to the dissolution testing of enteric coated products. Eur. J. Pharm. Biopharm. 78, 151-157.

Melocchi, A., Parietti, F., Maroni, A., Foppoli, A., Gazzaniga, A., Zema, L., 2016. Hot-melt extruded filaments based on pharmaceutical grade polymers for 3D printing by fused deposition modeling. International Journal of Pharmaceutics 509, 255-263.

Merchant, H.A., Frost, J., Basit, A.W., 2012. Apparatus and method for testing medicaments. PCT/GB2013/051145

Merchant, H.A., Goyanes, A., Parashar, N., Basit, A.W., 2014. Predicting the gastrointestinal behaviour of modified-release products: Utility of a novel dynamic dissolution test apparatus involving the use of bicarbonate buffers. Int. J. Pharm. 475, 585-591.

Okwuosa, T.C., Pereira, B.C., Arafat, B., Cieszynska, M., Isreb, A., Alhnan, M.A., 2017. Fabricating a Shell-Core Delayed Release Tablet Using Dual FDM 3D Printing for PatientCentred Therapy. Pharmaceutical Research 34, 427-437.

Okwuosa, T.C., Stefaniak, D., Arafat, B., Isreb, A., Wan, K.-W., Alhnan, M.A., 2016. A Lower Temperature FDM 3D Printing for the Manufacture of Patient-Specific Immediate Release Tablets. Pharmaceutical Research 33, 2704-2712.

Partee, B., Hollister, S.J., Das, S., 2006. Selective Laser Sintering Process Optimization for Layered Manufacturing of CAPA[sup $\AA] 6501$ Polycaprolactone Bone Tissue Engineering Scaffolds. Journal of Manufacturing Science and Engineering 128, 531.

Pietrzak, K., Isreb, A., Alhnan, M.A., 2015. A flexible-dose dispenser for immediate and extended release 3D printed tablets. European Journal of Pharmaceutics and Biopharmaceutics 96, 380-387. 
Rowe, C.W., Katstra, W.E., Palazzolo, R.D., Giritlioglu, B., Teung, P., Cima, M.J., 2000. Multimechanism oral dosage forms fabricated by three dimensional printing. Journal of controlled release : official journal of the Controlled Release Society 66, 11-17.

Shirazi, S.F.S., Gharehkhani, S., Mehrali, M., Yarmand, H., Metselaar, H.S.C., Adib Kadri, N., Osman, N.A.A., 2015. A review on powder-based additive manufacturing for tissue engineering: selective laser sintering and inkjet $3 \mathrm{D}$ printing. Science and Technology of Advanced Materials 16, 033502.

USP, 2017. Tablet Friability. http://www.usp.org/harmonization/tablet-friability.

Wang, J., Goyanes, A., Gaisford, S., Basit, A.W., 2016. Stereolithographic (SLA) 3D printing of oral modified-release dosage forms. Int J Pharm 503, 207-212.

Wu, B.M., Borland, S.W., Giordano, R.A., Cima, L.G., Sachs, E.M., Cima, M.J., 1996. Solid free-form fabrication of drug delivery devices. Journal of Controlled Release 40, 77-87.

Yalkowsky, S.H., He, Y., 2003. Handbook of aqueous solubility data. CRC Press, Boca Raton.

Yu, D.G., Branford-White, C., Ma, Z.H., Zhu, L.M., Li, X.Y., Yang, X.L., 2009. Novel drug delivery devices for providing linear release profiles fabricated by 3DP. Int J Pharm 370, 160-166.

Yu, D.G., Yang, X.L., Huang, W.D., Liu, J., Wang, Y.G., Xu, H., 2007. Tablets with material gradients fabricated by three-dimensional printing. J Pharm Sci 96, 2446-2456.

Yu, D.G., Zhu, L.M., Branford-White, C.J., Yang, X.L., 2008. Three-dimensional printing in pharmaceutics: Promises and problems. J. Pharm. Sci. 97, 3666-3690. 
Figure 1. Image of the printlets, on the top from left to right $\mathrm{K} 5, \mathrm{~K} 20, \mathrm{~K} 35$; at the bottom, from left to right E5, E20, E35.

Figure 2. X-ray micro-CT images of a quarter section of the printlets. On the top from left to right $\mathrm{K} 5, \mathrm{~K} 20, \mathrm{~K} 35$. On the bottom, from left to right E5, E20, E35.

Figure 3. SEM images of the printlets vertical sections, on the top from left to right $\mathrm{K} 5$, K20, K35. On the bottom, from left to right E5, E20, E35.

Figure 4. DSC thermograms of pure paracetamol, mainindividual polymers, mixtures before printing and printlets.

Figure 5. X-ray powder diffractograms of pure paracetamol, mixtures before printed and 3DP discs.

535

Figure 6. Drug dissolution profiles from Kollicoat printlets. Red line shows the pH values of the media.

Figure 7. Drug dissolution profiles from Eudragit printlets. Red line shows the $\mathrm{pH}$ values of 540 the media. 
Table 1. Printlets composition

\begin{tabular}{cccc}
\hline Formulation $^{*}$ & Kollicoat IR (\%) & Eudragit L100-55 (\%) & Paracetamol (\%) \\
\hline K5 & 92 & - & 5 \\
K20 & 77 & - & 20 \\
K35 & 62 & - & 35 \\
E5 & - & 92 & 5 \\
E20 & - & 77 & 20 \\
E35 & - & 62 & 35 \\
\hline
\end{tabular}

${ }^{*}$ All formulations contain 3\% w/w Candurin ${ }^{\circledR}$ Gold Sheen. 
Table 2. Physical properties of the printlets

\begin{tabular}{cccccc}
\hline Formulation & $\begin{array}{c}\text { Drug } \\
\text { loading }\end{array}$ & $\begin{array}{c}\text { Crushing } \\
\text { strength }(\mathrm{N})\end{array}$ & $\begin{array}{c}\text { Friability } \\
(\%)\end{array}$ & $\begin{array}{c}\text { Closed porosity } \\
(\%)\end{array}$ & $\begin{array}{c}\text { Open porosity } \\
(\%)\end{array}$ \\
\hline K5 & 4.9 & $>485$ & 0.02 & 0.2 & 33.3 \\
K20 & 20.4 & $>485$ & 0.08 & 0.4 & 24.6 \\
K35 & 35.7 & $>485$ & 0.13 & 0.8 & 24.0 \\
E5 & 5.0 & 284 & 0.56 & 0.1 & 29.3 \\
E20 & 20.1 & 285 & 0.31 & 1.0 & 20.3 \\
E35 & 35.3 & 414 & 0.11 & 4.7 & 1.0 \\
\hline
\end{tabular}



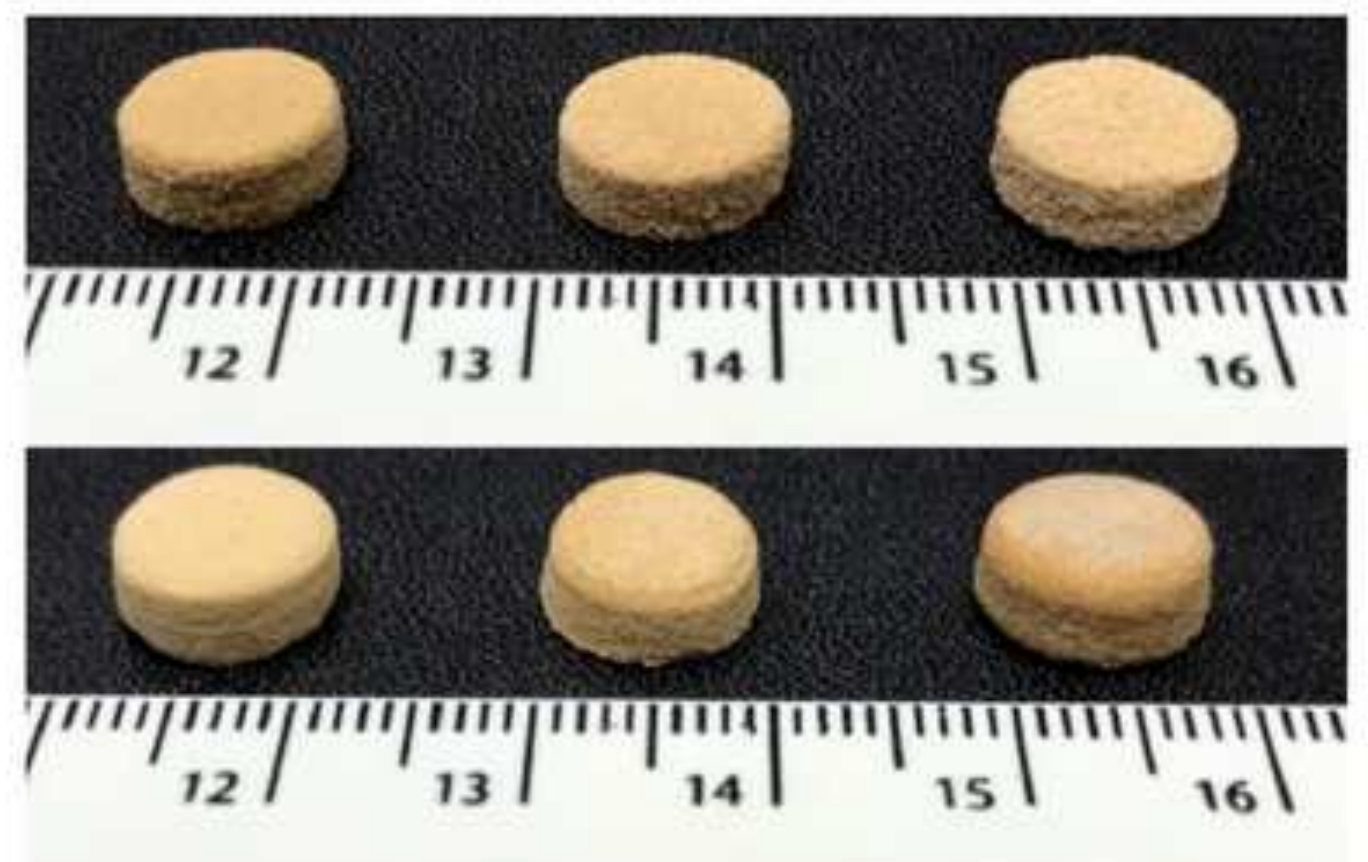

Figure 1

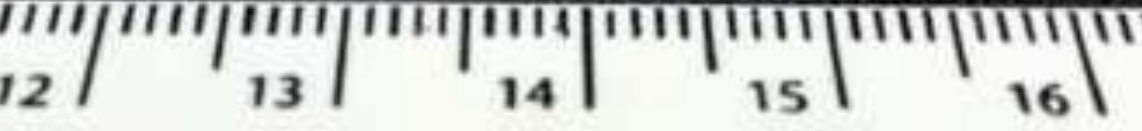

Figure 1
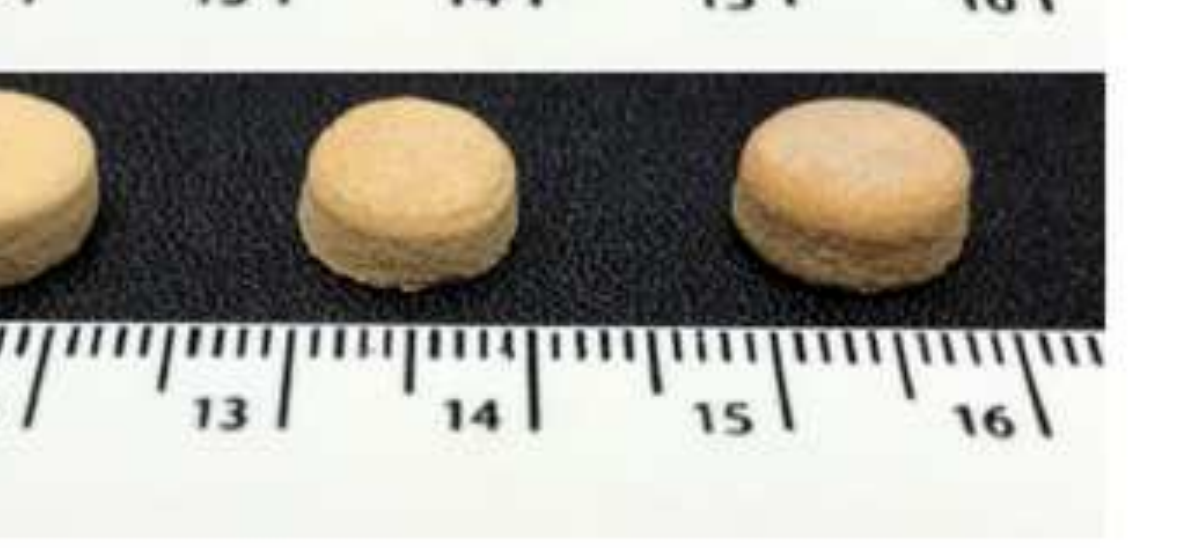

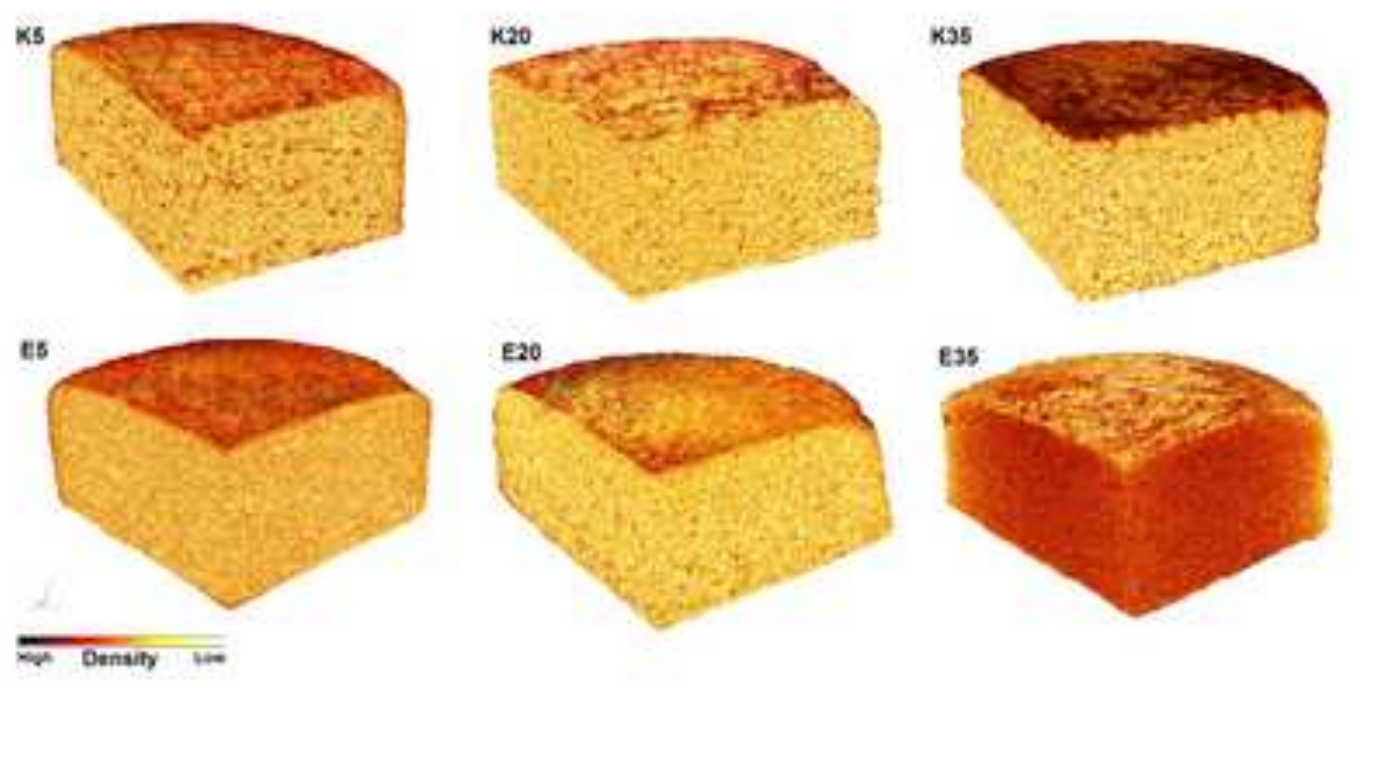

Figure 2

Figure 2

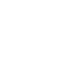
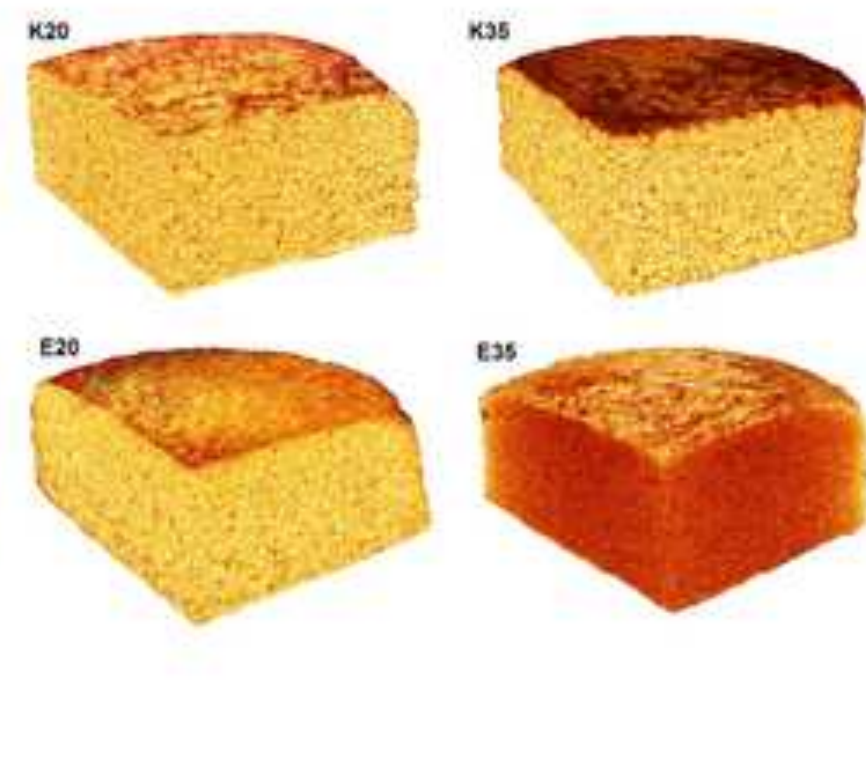

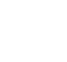

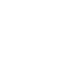

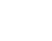
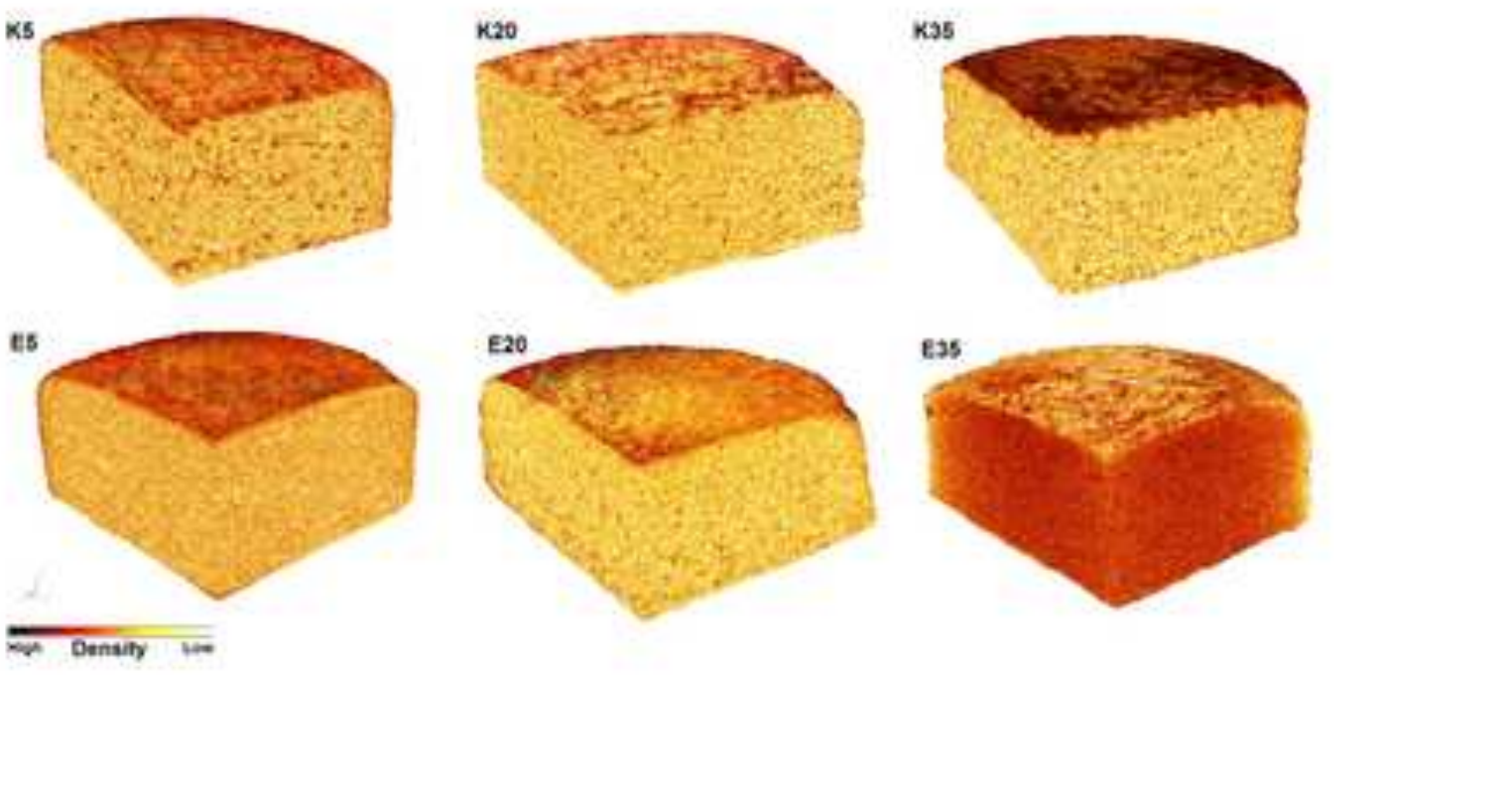

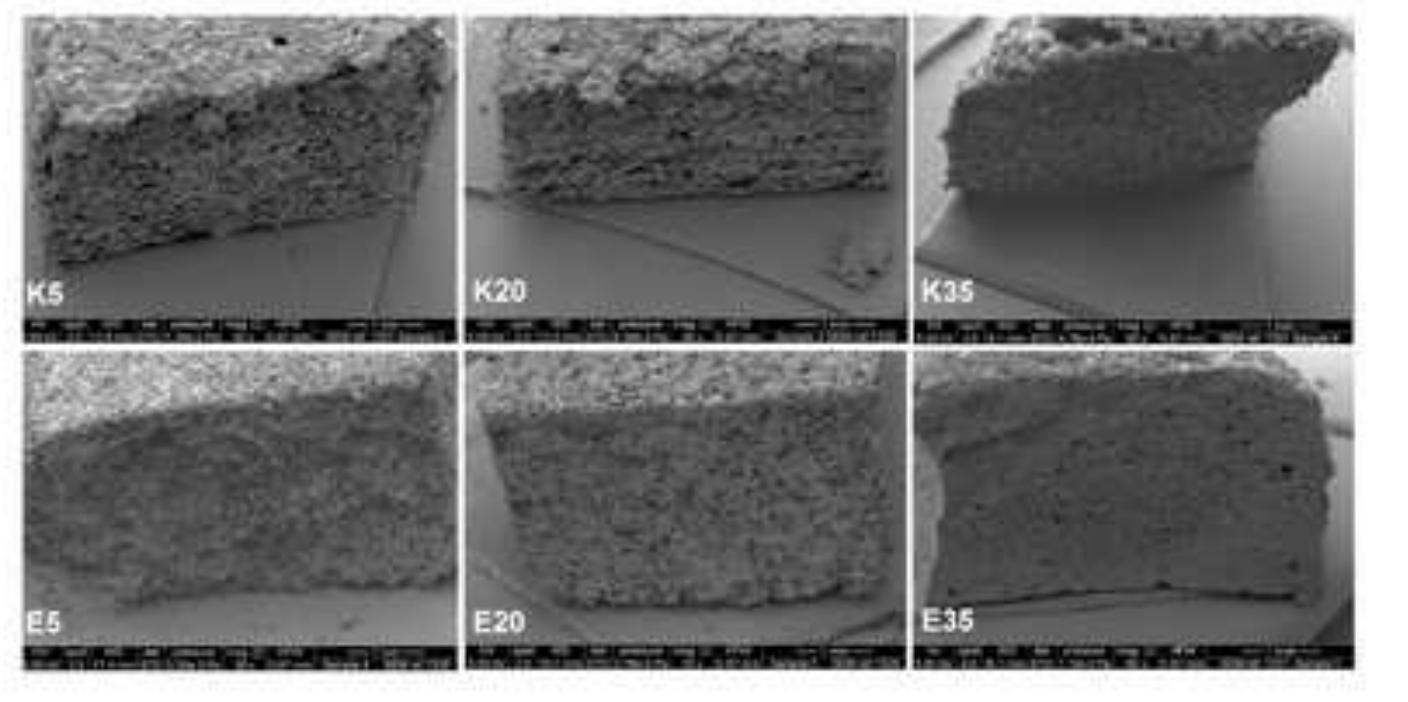

Figure 3 

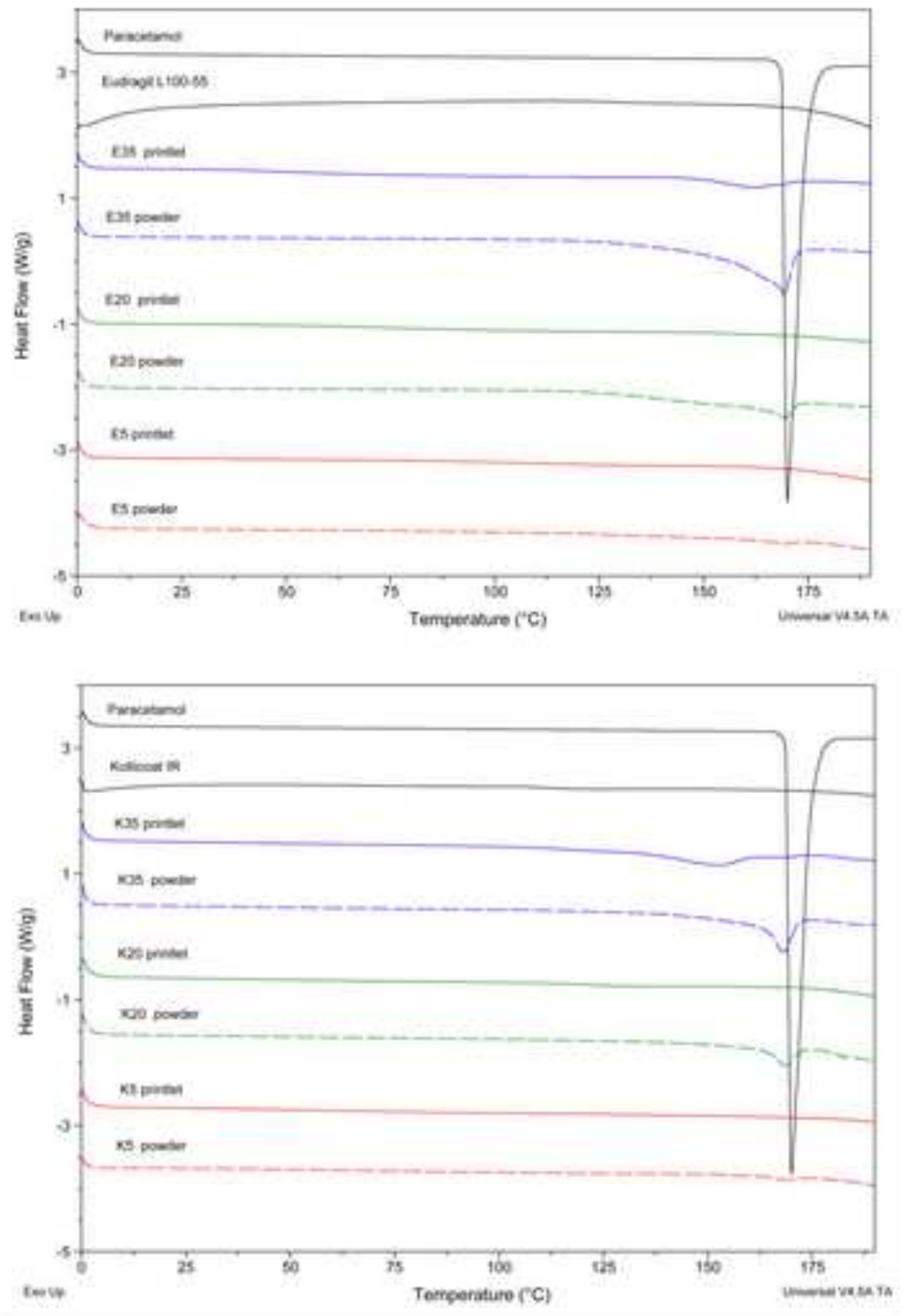

Figure 4

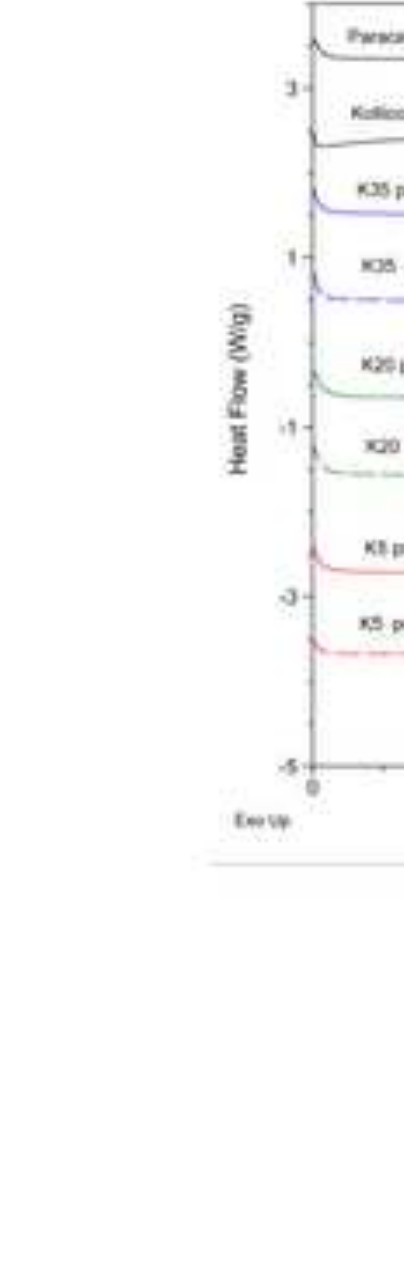

Figure 4

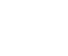

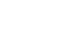

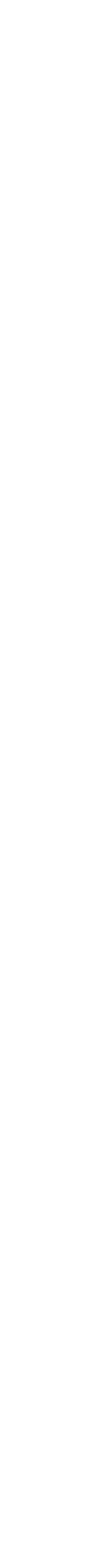




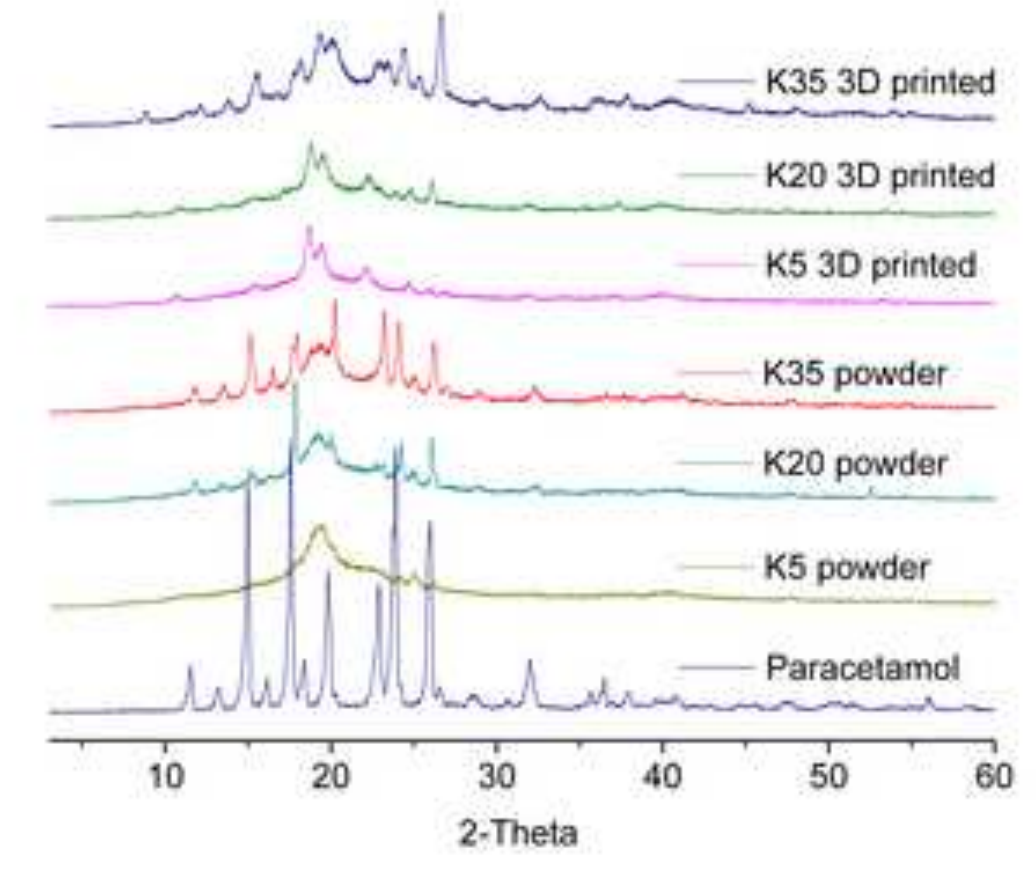

E35 3D printed

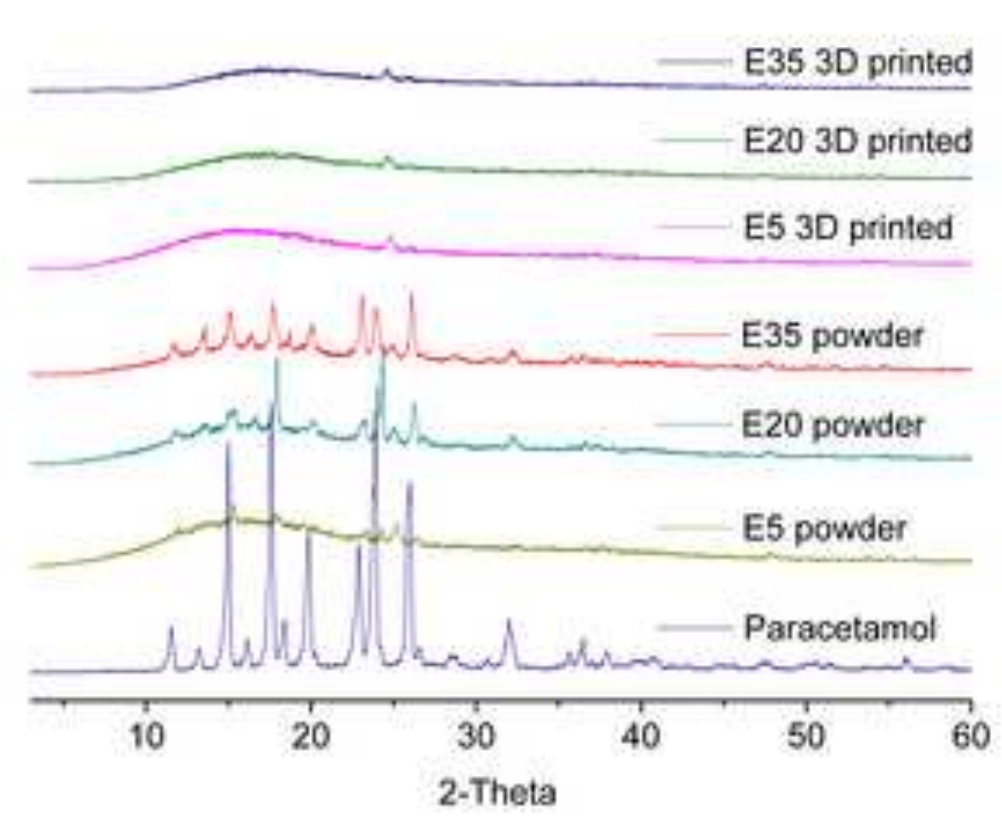

Figure 5 


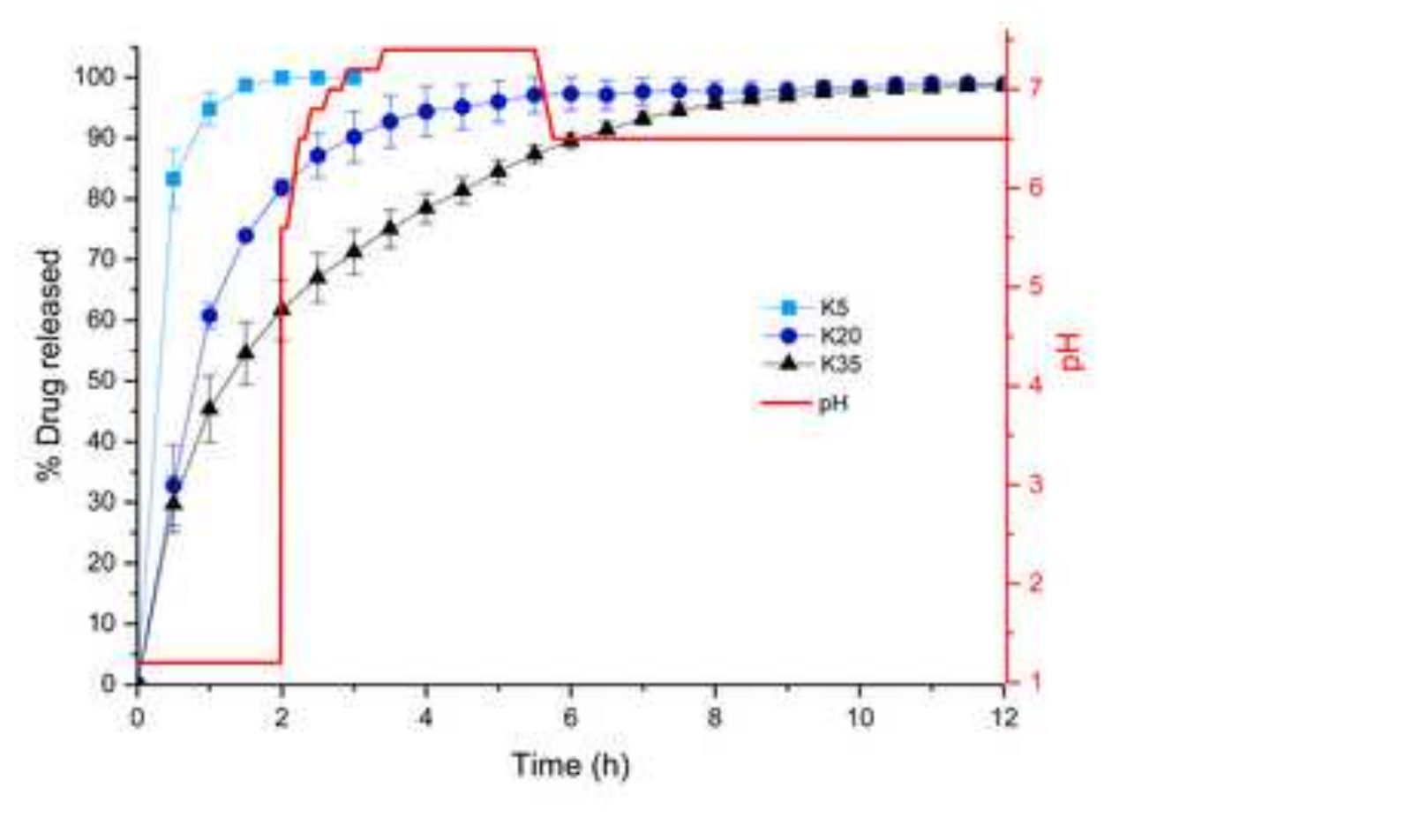

Figure 6

Time (h)

\section{Figure 6} 6

(1)

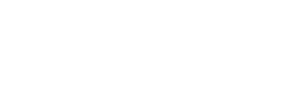

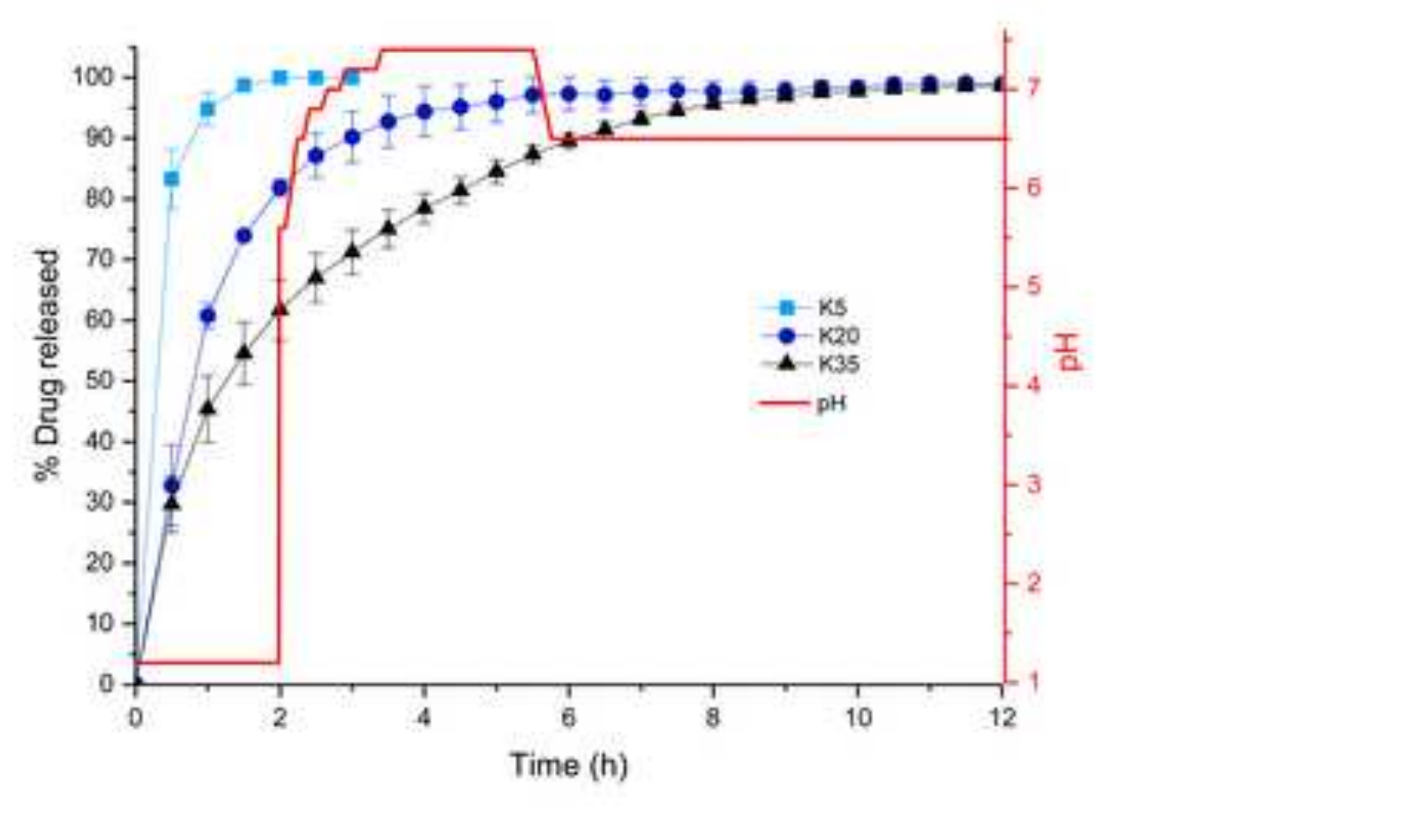

政

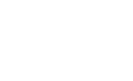


Figure 7
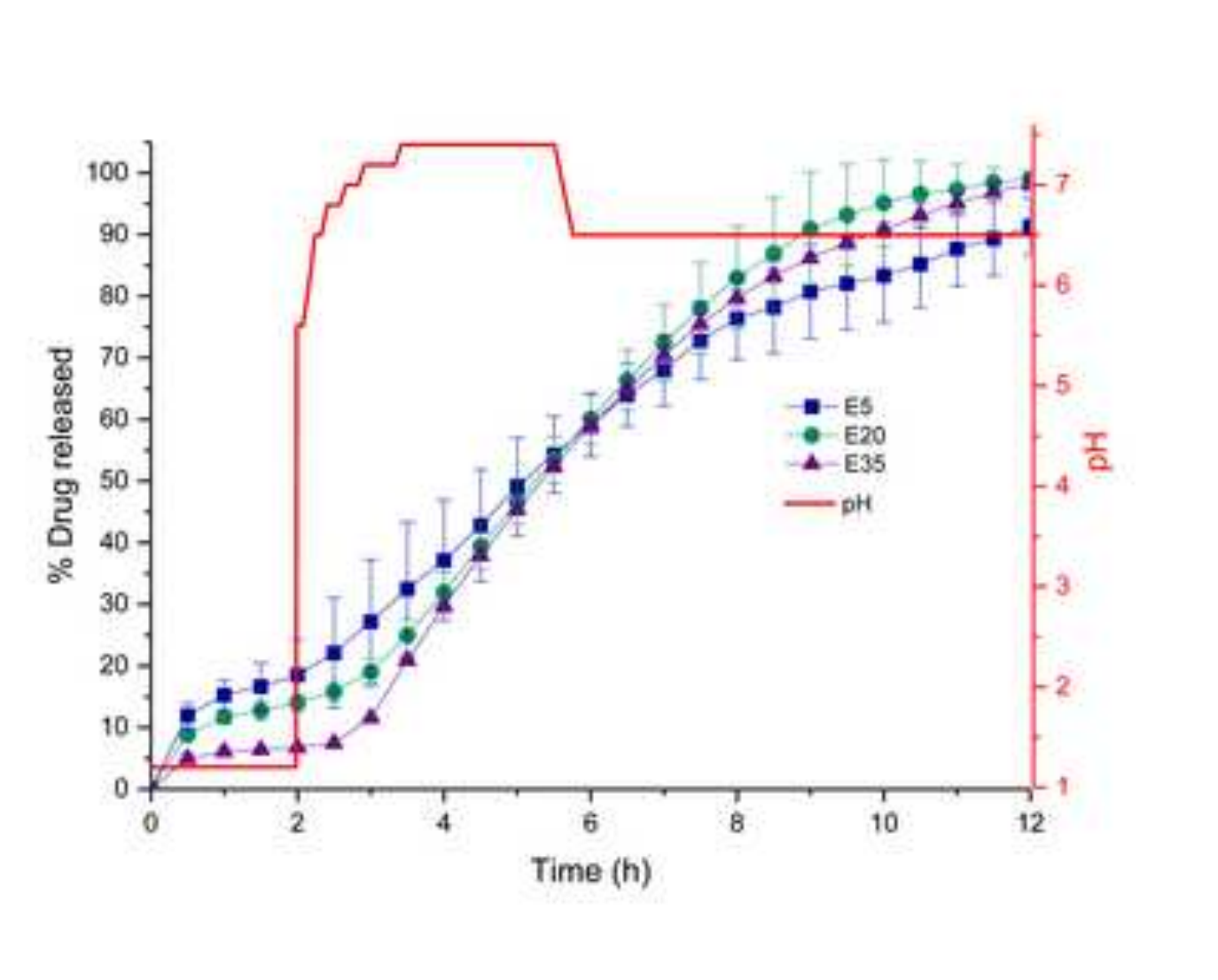

.
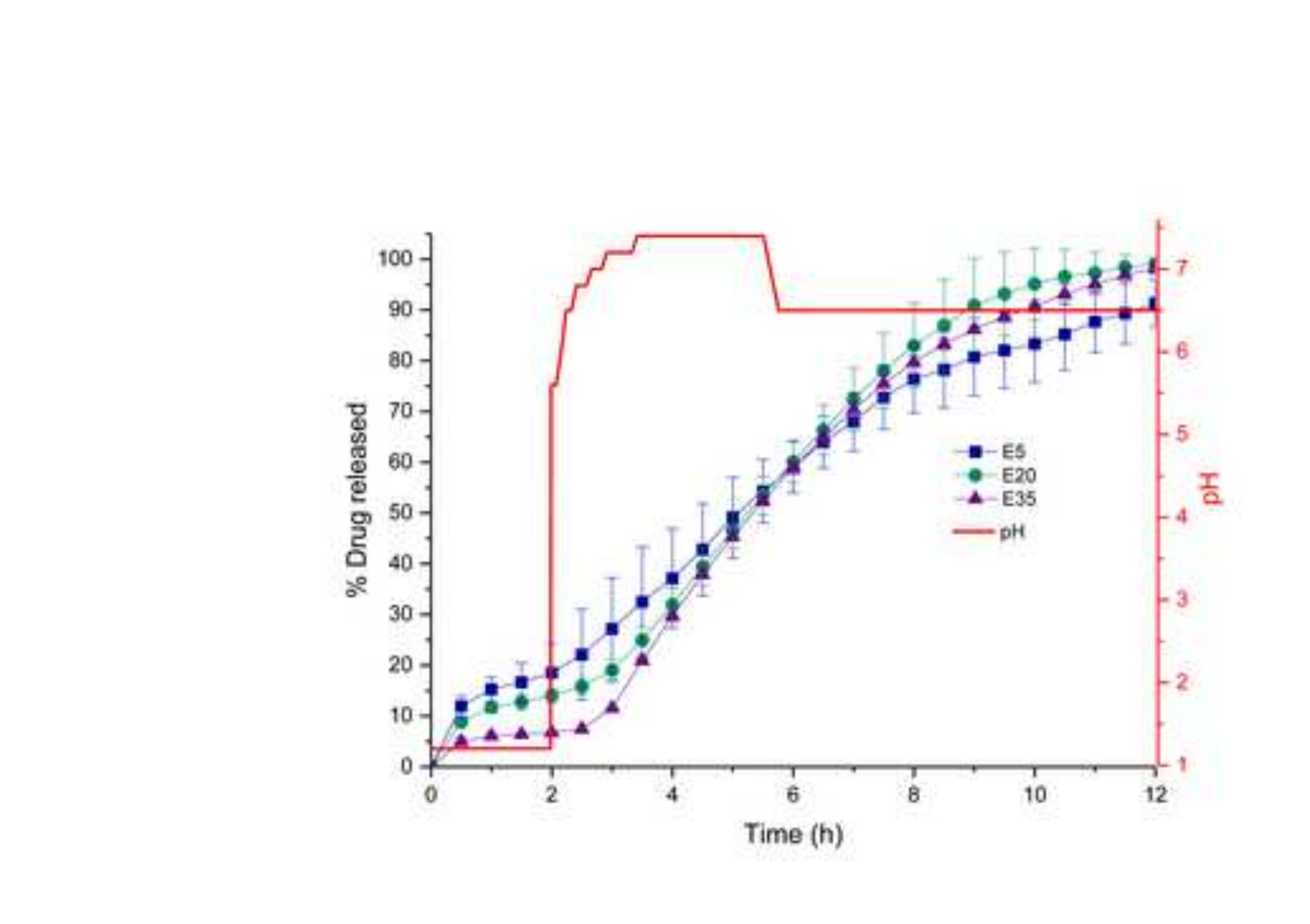

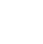

Time (h)

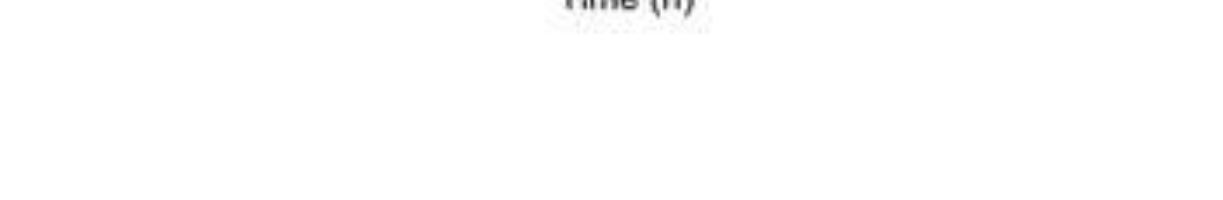

